\title{
Does the ERAS program reduce the metabolic stress in colorectal surgery? A comparative study.
}

\author{
Casado Neira J11, Sánchez Expósito P1, Molnar V11, Barbero Mielgo M¹, Sánchez-Cabezudo Moreno \\ $C^{1}$, García Fernández J1. \\ ${ }^{1}$ Hospital Puerta de Hierro de Majadahonda
}

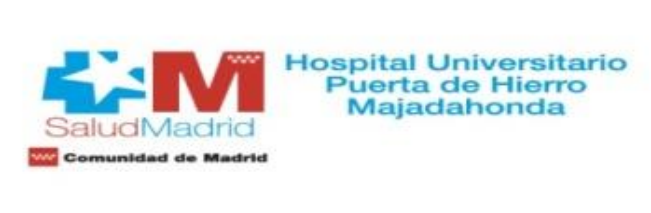

Background and Goal of Study: There are now many programs focused on clinical strategies that attenuate metabolic stress. In our hospital we are following the Enhanced Recovery After Surgery (ERAS) program in colorrectal surgery.. The aim of this study is to demonstrate a reduction of the metabolic response in colorectal surgery using the ERAS program in comparison with the cases recollected before the implementation of the program; using the glycemic control and the C-Reactive Protein(RCP) as predictors of metabolic stress.

Materials and Methods: We compared the RCP values and the subtraction between postoperative and basal glycemia on the first, third and fifth postoperative days after colorrectal surgery between two groups: The prospective group ( $n=133$, medium age $67.54 \pm 12.34$, CR-POSSUM 16 \pm 0.71 ) were subjected to colorrectal surgery in 2017 (POST-

ERAS). The retrospective group ( $n=37$, medium age 65.8 \pm 13.88 , CR-POSSUM 9.53 \pm 3.37 ), subjected to the same surgery before ERAS program. In order to compare the studied parameters we used the mean and standard deviation.

\section{Results and Discussion:}

In both situations we found a non-significant reduction on the level of the predictors we were studying.

\begin{tabular}{|c|c|c|c|}
\hline $\begin{array}{r}\text { GLYCEMIA } \\
\mathrm{mg} / \mathrm{d} /\end{array}$ & POST-ERAS & PRE-ERAS & REDUCTION \\
\hline 1st d-basal & $13.2 \pm 35$ & $20.9 \pm 44$ & -7.7 \\
\hline 3rd d -basal & $-5.2 \pm 77$ & $-2.8 \pm 31$ & -2.4 \\
\hline 5th $d$-basal & $-10.3 \pm 17$ & $-3.7 \pm 29$ & -6.6 \\
\hline CRP & & & \\
\hline 1st d & $63.3 \pm 41$ & $87.3 \pm 40$ & -24 \\
\hline $3 \mathrm{rd} d$ & $69.7 \pm 53$ & $93.4 \pm 64$ & -23.7 \\
\hline 5th $d$ & $45.4 \pm 49$ & $72.1 \pm 104$ & -26.7 \\
\hline
\end{tabular}
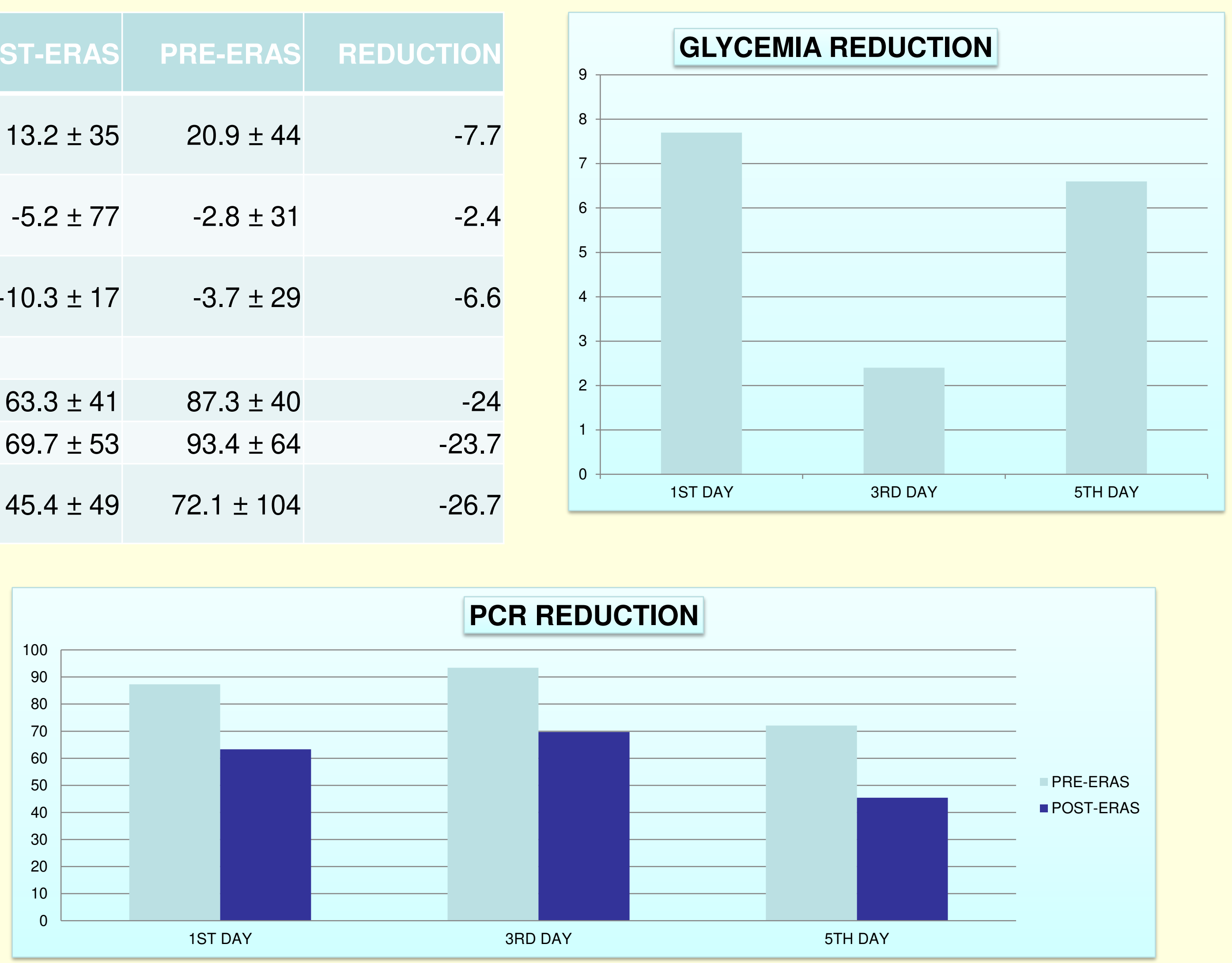

Conclusion(s): A reduction of the metabolic stress response, as shown by the reduction of the studied parameters, was observed. These results may highlight the significant role of ERAS program in maintaining homeostasis, therefore proving that the ERAS program reduces the metabolic response in colorectal surgery. Probably to achieve a significant result we should enlarge the clinical trial. 